\title{
Gap deformation and classical wave localization in disordered two-dimensional photonic band gap materials
}

\author{
E. Lidorikis ${ }^{1}$, M. M. Sigalas ${ }^{1}$, E. N. Economou ${ }^{2}$ and C. M. Soukoulis ${ }^{1,2}$ \\ ${ }^{1}$ Ames Laboratory-U.S. DOE and Department of Physics and Astronomy, Iowa State University, Ames, Iowa 50011 \\ ${ }^{2}$ Research Center of Crete-FORTH and Department of Physics, University of Crete, Heraklion, Crete 71110, Greece
}

\begin{abstract}
By using two $a b$ initio numerical methods we study the effects that disorder has on the spectral gaps and on wave localization in two-dimensional photonic band gap materials. We find that there are basically two different responses depending on the lattice realization (solid dielectric cylinders in air or vise versa), the wave polarization, and the particular form under which disorder is introduced. Two different pictures for the photonic states are employed, the "nearly free" photon and the "strongly localized" photon. These originate from the two different mechanisms responsible for the formation of the spectral gaps, ie. multiple scattering and single scatterer resonances, and they qualitatively explain our results.
\end{abstract}

PACS numbers:

\section{INTRODUCTION}

Electromagnetic waves traveling in periodic dielectric structures will undergo multiple scattering. For the proper structural parameters and wave frequencies, all waves may backscatter coherently; the result is total inhibition of propagation inside the structure. Such structures are called photonic band gap (PBG) materials 1 国 or photonic crystals, and the corresponding frequency ranges, for which propagation is not allowed, photonic band gaps or stop bands. PBG materials can be artificially made in one, two, or three dimensions. For example, a periodic lattice of dielectric spheres embedded in a different dielectric medium would work as a three-dimensional PBG material, for the proper choice of lattice symmetry, dielectric contrast, and sphere volume filling ratio. In two dimensions, a periodic array of parallel, infinitely long, dielectric cylinders could work as a two-dimensional PBG material, prohibiting propagation in a direction perpendicular to the cylinders' axis for some frequency range(s). The absence of optical modes in a photonic band gap is often considered as analogous to the absence of electronic energy eigenstates in the semiconductor energy gap. The ability of PBG materials to modulate electromagnetic wave propagation, in a similar way semiconductors modulate the electric current flow, can have a profound impact in many areas in pure and applied physics. It is then of fundamental importance to study the effects of disorder 3 t on the transmission properties of such materials.

Besides the non-resonant, macroscopic Bragg-like multiple sfattering, there is also a second, resonant mechanism, that contributes to the formation of the spectral gaps. This is 5 t the excitation of single scatterer Mie resonances $\mathrm{G}$. In a previous publication it was shown that for two-dimensional PBG materials, for the $E$ polarization scalar wave case (electric field parallel to the cylinders' axis), these Mie resonances are analogous to the electronic orbitals in semiconductors. The idea of the linear combination of atomic orbitals (LCAO) method was extended to the classical wave case as a linear combination of Mie resonances (LCMR), leading to a successful tight-binding (TB) parameterization for photonic band gap materials. This moves the picture for the photon states, from a one analogous to the nearly free electron model, to the one analogous to the strongly localized electron whose transport is achieved only by hopping (tunneling) from atom to atom. Depending then on which mechanism is dominant for the formation of the photonic gaps, we expect different changes to the system's properties when disorder is introduced. If the Bragg-like multiple scattering mechanism is the dominant one, the photonic gaps should close quickly with increasing disorder, while if it is the excitation of Mie resonances, the photonic gaps should survive large amounts of disorder, in a similar way the electronic energy gap survives in amorphous silicon.

In this paper we will use two ab initio numerical methods to study the effects of disorder on photonic gap formation and wave localization in two-dimensional PBG materials. The first is the finite difference time domain (FDTD) spectral method 10 , from which we obtain the photonic density of states for an infinite, disordered PBG material, and the second is the transfer matrix technique 11 , from which we obtain the transmission coefficient for a wave incident onto a finite slab of the disordered PBG material. From the transmission coefficient we can obtain the localization length for the photonic states of the disordered materialt. The study will be on both PBG material realizations (solid high dielectric cylinders in air and cylindrical air holes in high dielectric), for both wave polarizations, and it will incorporate three different disorder realizations: disorder in position, radius, and dielectric constant (these systems, though, will still be periodic on the average). We will find that only the case of solid dielectric cylinders in air with 
the wave $E_{z}$-polarized exhibits the behavior expected from the strongly localized photon picture, while for all other cases, the nearly free photon picture seems to be the dominant one.

\section{NUMERICAL METHODS}

Electromagnetic wave propagation in lossless composite dielectric media is described by Maxwell's equations

$$
\mu \frac{\partial \vec{H}}{\partial t}=-\vec{\nabla} \times \vec{E}, \quad \epsilon(\vec{r}) \frac{\partial \vec{E}}{\partial t}=\vec{\nabla} \times \vec{H},
$$

where the dielectric constant $\epsilon(\vec{r})$ is a function of position. In two dimensions, the two independent wave polarizations are decoupled. We assume the variation of the dielectric constant, as well as the propagation direction, along the $x y$ plane, and so, the cylinders along the $z$ axis. One of the polarizations is with the electric field parallel to the $z$ axis and the magnetic field on the $x y$ plane $\left(E_{z}\right.$ or TM polarized) and obeys a scalar wave equation. The other one with the magnetic field parallel to the $z$ axis and the electric field on the $x y$ plane ( $H_{z}$ or TE polarized) and obeys a vector wave equation.

The first method we will use, to study disordered PBG materials, is the FDTD spectral method 12.13. In our FDTD scheme, we first discretize the $x y$ plane into a fine uniform grid. Each grid point is centered in a unit cell which is further discretized into a $10 \times 10$ subgrid, on which an arithmetic average of the dielectric constant is performed. In our problem we will assume dispersionless and lossless materials. For the $E_{z}$ polarization case we define the electric field on this grid and the magnetic field on two additional grids, one tilted by $(d / 2,0)$, on which $H_{y}$ is defined, and one tilted by $(0, d / 2)$, on which we define $H_{x} . d$ is the side of the grid cell. The corresponding finite-difference equations for the space derivatives that are used in the curl operators are then central-difference in nature and second-order accurate. The electric and magnetic fields are also displaced in time by a half time step $\Delta t / 2$, resulting in a "leapfrog" arrangement and central-difference equations for the time derivatives as well. If one initialize the electric and magnetic fields at $t=t_{0}$ and $t=t_{0}+\Delta t / 2$ respectively, then updating the values of the electric field for each grid point $(i, j)$ at $t=t_{0}+\Delta t$ is done by

$$
\begin{aligned}
\left.E_{z}\right|_{i, j} ^{t_{0}+\Delta t}=\left.E_{z}\right|_{i, j} ^{t_{0}} & +\frac{\Delta t}{d \epsilon_{i, j}}\left(\left.H_{y}\right|_{i+1 / 2, j} ^{t_{0}+\Delta t / 2}-\left.H_{y}\right|_{i-1 / 2, j} ^{t_{0}+\Delta t / 2}-\right. \\
& \left.-\left.H_{x}\right|_{i, j+1 / 2} ^{t_{0}+\Delta t / 2}+\left.H_{x}\right|_{i, j-1 / 2} ^{t_{0}+\Delta t / 2}\right)
\end{aligned}
$$

where $\epsilon_{i, j}$ is the averaged dielectric constant for the grid point $(i, j)$. Similar equations follow for updating the magnetic field components at $t=t_{0}+3 \Delta t / 2$, then again Eq. (2) for $E_{z}$ at $t=t_{0}+2 \Delta t$ etc. This way the time evolution of the system can be recorded. For numerical stability and good convergence the number of grid points per wavelength $\lambda / d$ must be at least 20, and also $\Delta t \leq d / \sqrt{2} c$, where $c$ the speed of light in vacuum. Similar equations, with the roles of the electric and magnetic fields interchanged, apply for the $H_{z}$ polarization case.

In order to find the eigenmodes of a particular periodic (or disordered) system, we first initialize the electric and magnetic fields in the unit cell (or a suitable supercell) using periodic boundary conditions: $\vec{E}(\vec{r}+\vec{a})=e^{i \vec{k} \vec{a}} \vec{E}(\vec{r})$ and similarly for $\vec{H}(\vec{r})$, where $\vec{k}$ is the corresponding Bloch wave vector and $\vec{a}$ the lattice vector. These fields must have nonzero projections with the modes in search. We choose a superposition of Bloch waves for the magnetic field and set zero the electric field:

$$
\vec{H}(\vec{r})=\sum_{\vec{g}} \hat{v}_{\vec{g}} e^{i(\vec{k}+\vec{g}) \vec{r}+i \phi_{\vec{g}}}, \quad \vec{E}(\vec{r})=0
$$

where $\phi_{\vec{g}}$ is just a random phase and the unit vector $\hat{v}$ is perpendicular to both $\vec{E}$ and $(\vec{k}+\vec{g})$, ensuring that $\vec{H}$ is transverse and that $\vec{\nabla} \vec{H}=0$. Once the initial fields are defined, we can evolve them in time using the "leapfrog" difference equations, while recording the field values as a time series for some sampling points. As the electric fields "builds" up, some particular modes dominate while most are depressed, reflecting the underline lattice symmetries. Here we record only the $E_{z}$ field for the $E_{z}$ polarization case, and the $H_{z}$ field for the $H_{z}$ polarization. At the end of the simulation, the time series are Fourier transformed back into frequency space, and the eigenmodes $\omega(\vec{k})$ of the system appear as sharp peaks. The length of the simulation determines the frequency resolution while the time difference between successive recordings determines the maximum frequency considered. This method scales linearly with size: a larger system will still need the samenumber of time steps for the same frequency resolution, thus sometimes referred to also as an "order-N" method 12. 
Here we will use this method to obtain the system's density of states (DOS). If one chooses a large supercell instead of the unit cell, then for each $\vec{k}$ point inside it's first Brillouin zone, the Fourier transformed time series will consist of a number of peaks. Adding all contributions from all $\vec{k}$ 's will result to a smooth function for the DOS. This is in contrast to older methods that where using random fields as initial boundary conditions 1 . Random initial fields will ensure the condition for nonzero projections to all of the system's eigenmodes, but in order to get coupled with them during "built" up, a large simulation time is required. Furthermore, the produced DOS is not a smooth function of frequency, still consisting of a large collection of peaks, and thus being useful only as an indication for the existence of spectral gaps. In our method, the underline symmetries of the modes are already in the initial fields and so they couple easier with them. Also, the larger the supercell, the smaller is its first Brillouin zone, and so the smaller the frequencies we initialize through the various $\vec{k}$. This is why we can get smooth results even for very low frequencies. In Figs. 1 and 2, we show the calculated density of states for the case of solid dielectric cylinders in air and cylindrical air holes in dielectric respectively, both for a square lattice arrangement, and for both polarizations. Along with them we also plot the corresponding band structure as obtained with the plane wave expansion method. Our study is going to be based on these two photonic structures.

The second method we will use is the transfer matrix technique in order to obtain the transmission coefficient for a wave incident along the $x y$ plane on a slab (or a slice) of the photonic material. The slice is assumed uniform along the $z$ axis, and periodic along the $x$ direction through application of periodic boundary conditions, while in the $y$ direction it has a finite width $L$. In this method one first constructs the transmitted waves at one side of the slice and then integrates numerically the time-independent Maxwell's equations to the other side. There, the waves are projected into incident and reflected waves, and so a value for the transmission coefficient $T$ can be obtained. Here, we are interested in the wave localization in disordered photonic band gap materials, and in particularly in the localization length $\ell \sim-2 L / \ln T$.

A few remarks about the results of this method are in order. Waves with different incidence directions will have different reflection and transmission coefficients, so if one is looking for an average transmission, all directions should be included. It is shown, however, that there is also a large dependence on the surface plane along which the structure is cut. More specifically, a wave normally incident on a $(1,0)$ surface will have different transmission characteristics than a wave incident with $45^{\circ}$ on a $(1,1)$ surface. This is because certain modes can not always get coupled with the incident wave. One should then also average for the two different surface cuts, otherwise it will not be a true average. This is shown in Figs. 3 and 4 where we plot the $(1,0)$ and the $(1,1)$ cuts, each with both incidence directions (normal and $45^{\circ}$ with respect to the surface) averaged. We see that taken individually, none of them corresponds to the true gaps as shown in Figs. 1 and 2, but rather, to wider and generally displaced gaps. For example, in the $E_{z}$ polarization case in the first spectral gap (Figs. 3a and 4a), with the $(1,0)$ cut, the incident waves fail to couple with the the $\mathbf{M}$ modes of the first band, while with the $(1,1)$ cut, the incident waves fail to couple with the $\mathbf{X}$ modes of the second band.

This is expected to be lifted once disorder is introduced into our system, since the sense of direction will be somehow lost. Disorder can be introduced as a random displacement, a random change in the radius, or, a random change in the dielectric constant of the cylinders. It is not clear however what amount of disorder would be needed for this. We repeated the calculations for small enough amounts of disorder so that the spectral gaps, as found from the FDTD method, remain almost unchanged, for all three different disorder mechanisms. As seen in Figs. 3 and 4, indeed, in some cases the coupling is achieved. For example, for the first gap in the $E_{z}$-polarization case, with the $(1,0)$ cut, the $\mathbf{M}$ modes of the first band are now coupled with the incident waves and appear in the transmission diagram. These could be easily mistaken for disorder-induced localized states entering the gap, but they are not, since for the values of disorder used, the first gap is virtually unchanged. On the other hand, with the $(1,1)$ cut, the coupling to $\mathbf{X}$ modes of the second band is not yet achieved, still yielding a wrong picture for the gap. Increasing the disorder further will eventually destroy any sense of direction and there will be no distinction between the two cases. Figs. 3 and 4 will be useful as a guide of which results can be trusted and which can not, if one uses only one surface cut and small values of disorder. As a general rule, we can deduce that the $(1,0)$ cut should be used for the $E_{z}$ polarization case, while the $(1,1)$ cut would be better for the $H_{z}$ polarization case.

\section{RESULTS AND DISCUSSION}

We first looked into the spectral gaps' dependence on disorder using the FDTD spectral method. Our system consisted of a $8 \times 8$ supercell, each cell discretized into a $32 \times 32$ grid. We studied two systems: a square lattice array of solid cylinders, with dielectric constant $\epsilon_{a}=10$, in air $\left(\epsilon_{b}=1\right)$ with a filling ratio $f=0.28 \%$, and a square lattice array of air cylinders $\left(\epsilon_{a}=1\right)$ in dielectric material $\epsilon_{b}=10$, with air filling ratio $f=0.71 \%$, as described in Figs. 1 and 2 . We divided the supercell's first Brillouin zone into $10 \times 10$ grid, which for the irreducible part yields 66 different $\vec{k}$ points. 
For each particular disorder realization (i.e. disorder type) and disorder strength, we run the simulation for all these $66 \vec{k}$ 's. At each $\vec{k}$ however we use a different disordered configuration (i.e. a different seed in the random number generator), and so a large statistical sample is automatically included in our result In $^{2}$ each case the effective disorder is measured by the rms error of the dielectric constant $\langle\epsilon\rangle$, which is defined as 22.

$$
\epsilon^{2}=\frac{1}{N} \sum_{i=1}^{N}\left(\epsilon_{i}^{d}-\epsilon_{i}^{p}\right)^{2},
$$

where the sum goes over all $N=8 \times 8 \times 32 \times 32=65536$ grid points, $\epsilon_{i}^{d}$ and $\epsilon_{i}^{p}$ are the dielectric constants at site $i$ in the disordered and periodic case respectively, and $\langle\ldots\rangle$ means the average over different configurations (different $\vec{k}$ 's in our case). In both settings (dielectric cylinders in air and vise versa) the filling ratio of the high dielectric material is similar, and so $\langle\epsilon\rangle$ is expected to have the same meaning and weight.

Four different disorder realizations are studied: 1) disorder in position, without though allowing any cylinders to overlap with each other, 2) disorder in position allowing cylinder overlapping to occur, 3) disorder in radius, and 4) disorder in dielectric constant (the last one only in the solid cylinder case). For each different realization we consider various disorder strengths, and thus different effective disorders $\langle\epsilon\rangle$, for which we record the upper and lower gap edges for the first two photonic band gaps (if they exist). Results are summarized in Figs. 5 and 6, for the solid and air cylinder cases respectively. We note that the $E_{z}$ polarization case for the solid cylinders is very different from all other cases: the gaps survive very large amounts of positional disorder, especially if no overlaps are allowed. In fact, once the disorder becomes large enough for overlaps to be possible, the gap quickly closes, as shown in Fig. 5. The actual DOS graphs for the two different realizations of the positional disorder are shown in Fig. 7, for three different values of the effective disorder. On the other hand, if the disorder is of the third or fourth kind, the gaps close very quickly, even for modest values of the effective disorder.

The picture is very different in the other cases, as seen in Fig. 6. The effect of the positional disorder is the same, independent of whether overlaps are allowed or not. This is most clearly seen in Fig. 8, where the actual DOS graphs are plotted for the air cylinder case for both polarizations, and for both positional disorder realizations. Allowing the air cylinders to overlap, though, means that the connectivity of the background material will break. Our results, thus, indicate that there is no connection between the connectivity of the background material and the formation of the spectral gaps in this 2D case. Most importantly, however, we note that the disorder in radius has a similar effect with that of the positional disorder in closing the gaps. In fact, it is also similar to the effect of the disorder in radius for the $E_{z}$-solid-cylinder case. So, in the case of air cylinders, the type of the disorder that is introduced into the system does not play a significant role, but rather, it is only the effective disorder (measured through the dielectric constant's error function) that determines the effect on the spectral gaps. On the other hand, for the $E_{z}$-solid-cylinder case, the type of disorder plays a profound role: if the "shape" of the individual scatterer is preserved, the gaps can sustain large amounts of disorder, while if it is not preserved, the gaps collapse in a manner similar to the air cylinder case.

We next go over the localization length results, which were obtained with the transfer matrix technique. Here, our system consisted of a $3 \times 7$ supercell ( 3 along the $x$ axis), with each cell discretized into a $18 \times 18$ grid (a small supercell was used in order to ease the computation burden). In the $x$ direction we applied periodic boundary conditions, while in the $y$ direction the supercell was repeated 4 times, to provide a total length $L$ for the slab of $L=28$ unit cells. The structures studied are exactly the same as described before. The lattice was cut along one only symmetry direction, the $(1,0)$, since for large disorders we expect all "hidden" modes to be coupled with the incident wave (in any case, we know from Figs. 3 and 4 which results can be completely trusted and which can not). For each disorder realization and strength, we used 11 different $\vec{k}$ values uniformly distributed between normal and $45^{\circ}$ angle incidence, and for each $\vec{k}$ we used a different disordered configuration, so these will constitute our statistical sample. For each $\vec{k}$ we find the minimum transmission coefficient inside each gap, from which we find the minimum localization length, and then average over all $\vec{k}$ 's, ie. $\ell \sim-2 L /<\ln T>$ (in the periodic case we first averaged over $T$ in order to correctly account for different propagation directions, but in the highly disordered case it is not so much important any more, and so we just average over the localization lengths).

Our results are shown in Figs. 9 and 10 (because of the small statistical sample and the small supercell used, the data points appear very "noisy", especially for large disorders). We note here, as well, the distinct difference between the $E_{z}$-solid-cylinder case for positional disorder and all other cases. Especially for the first spectral gap, the localization length not only remains unaffected by the disorder, but it even decreases (this is not an artifact of the averaging procedure). The first conclusion from this, is that the mechanisms responsible for the gap formation in this case are unaffected by the presence of positional disorder, and so they are definitely not macroscopic (long-range) in nature. The fact that the localization length decreases, is attributed to the coupling of more $[1,1]$ symmetry modes with the incident wave as the disorder increases (they provide a smaller $\ell$ to the average, as seen in Fig. 3a). This decrease should not be mistaken for additional localization induced by the disorder (the classical analog of Anderson 
localization in electrons), since the latter is macroscopic in nature, and does not apply for strongly localized waves. The decrease in the localization length continues until a fairly large disorder value, and then it increases to a saturation value (the dielectric error function can reach only up to some value for positional disorder). This saturation value is higher for the case where overlaps are allowed, but still is very small compared to other cases, so waves remain strongly localized.

All other cases, on the other hand, show a common pattern of behavior: photon states become quickly de-localized with increasing disorder. The localization length is increased, until the point where the localization induced by the disorder becomes dominant. After this it starts decreasing, until finally it reaches some saturation point. Note also that there is an almost quantitative agreement between some cases that was not really expected, eg. for the disorder in radius in the first gap with the wave $E_{z}$-polarized, for both lattice settings, as seen in Figs. 9a and 10a. Only the case of disorder in the dielectric constant seems to deviate, having very quickly a very large effect, with the localization length directly saturating to some constant value. So, for air cylinders in dielectric with any type of disorder, and for the $E_{z}$-solid-cylinder case with disorder that does not preserve the scatterer's "shape", the behavior under disorder is similar.

All these results can be understood if we adopt two different "pictures" for the photon states, depending on which is the dominant mechanism that is responsible for the formation of the spectral gaps in each case. The first is the "nearly free" photon picture, in which the gap forming mechanism is the non-resonant macroscopic Bragg-like multiple scattering, while the second is the "strongly localized" photon picture, in which the gap forming mechanism is the microscopic (short-range) excitation of single scatterer Mie resonances.

Sharp Mie resonances appear only for the solid cylinder case, and they can be thought as analogous to the atomic orbitals in semiconductors. Using this analogy, a tight-binding model, based on alinear combination of Mie resonances, was recently developed for the photonic states in the $E_{z}$-solid-cylinder caset. But if a tight-binding model can give a satisfactory description of the photonic states, then it is expected that certain behavioral patterns found in semiconductors should apply in our case too. So, positional disorder should have a small effect on the gaps, in a similar way the energy gap survives in amorphous silicon. Also, changing the scatterer should have a similar effect as changing the atoms in the semiconductor, yielding a large amount of impurity modes that quickly destroys the gap. This pattern is definitely confirmed here for the $E_{z}$-solid-cylinder case. In this case, multiple scattering and interference can only help to make the gaps wider, but are definitely not decisive on the existence of a gap.

For the macroscopic Bragg-like multiple scattering mechanism, the lattice periodicity is a very important factor for the existence of a spectral gap. If it is destroyed, then coherence in the backscattered waves will be destroyed, and so will the spectral gaps. It is of small consequence the exact way that the periodicity is destroyed, and so different disorder realizations will have similar effects. Also, since the gaps close more easily, it will be easier to observe the localization induced on the waves by the disorder itself, ie. the classical analog of Anderson localization in electrons. All these are recognized in the case of air cylinders in dielectric.

Finally, in the $H_{z}$-solid-cylinder case, there were no gaps to begin with, and so we can have no results about it. However, sharp Mie resonances appear for this case as well, and if their excitation was the dominant scattering mechanism, a gap would be expected here as well. The difference with the $E_{z}$ is that the former is described by a vector wave equation, while the latter by a scalar one (and thus closer to the electronic case). The form of the wave equation must, then, be an important factor in determining the relative strength of the two gap forming mechanisms.

\section{CONCLUSIONS}

We have shown that several results in periodic and random photonic band gap materials can be understood in terms of two distinct photonic states: (a) The "local" states, based on a single scatterer Mie resonance, with the multiple scattering playing a minor role; these states are more conveniently described in terms of an LCAO-type of approach and are the analog of the $d$-states in transition metals. "Local" photonic states appear in the case of high dielectric cylinders surrounded by a low-dielectric host and for E-polarized waves. (b) The "nearly free" photonic states, where Bragg-like multiple scattering is the dominant mechanism responsible for their appearance; these states are more conveniently described in terms of a pseudopotential-type of approach and are the analog of $s$ (or $p$ ) states in simple metals.

Each type of photonic states responds differently to the presence of disorder: For the "local" states case, the gap is robust as the periodicity is destroyed, and it is hardly affected by the disorder as long as the identity of each individual scatterer is preserved; however, if the shape, or other characteristics influencing the scattering cross section of each individual scatterer, is altered by disorder, the gap tends to disappear. On the other hand, for the "nearly free" states case, the gap is very sensitive and tends to disappear easily as the periodicity is destroyed. 


\section{ACKNOWLEDGMENTS}

Ames Laboratory is operated for the U. S. Department of Energy by Iowa State University under contract No.

W-7405-ENG-82. This work was supported by the Director of Energy Research office of Basic Energy Science and Advanced Energy Projects. It was also supported by the Army Research Office, a E.U. grant, and a NATO grant.

${ }^{1}$ For excellent reviews on photonic band gap materials see the proceedings of the NATO ARW, Photonic Band Gaps and Localization, ed. by C. M. Soukoulis, (Plenum, N.Y., 1993); Photonic Band Gap Materials, ed. by C. M. Soukoulis, NATO ASI, Series E, vol. 315.

${ }^{2}$ J. D. Joannopoulos, R. D. Meade, and J. N. Winn Photonic Crystals (Princeton University Press, Princeton, 1995).

${ }^{3}$ S. Fan, P. R. Villeneuve, and J. D. Joannopoulos, J. Appl. Phys. 78, 1415 (1995).

${ }^{4}$ M. M. Sigalas, C. M. Soukoulis, C. T. Chan, and D. Turner, Phys. Rev. B 53, 8340 (1996); M. M. Sigalas, C. M. Soukoulis, C. T. Chan, and K. M. Ho in Photonic Band Gap Materials, p. 563, ed. by C. M. Soukoulis (Kluwer, Dordrecht, 1996).

${ }^{5}$ S. Datta, C. T. Chan, K. M. Ho, C. M. Soukoulis, and E. N. Economou, in Photonic Band Gaps and Localization [Ref. [1](a)], p. 289.

${ }^{6}$ M. Kafesaki, E. N. Economou, and M. M. Sigalas, in Photonic Band Gap Materials [Ref. [1](c)], p. 143.

${ }^{7}$ E. Lidorikis, M. M. Sigalas, E. N. Economou, and C. M. Soukoulis, Phys. Rev. Lett. 81, 1405 (1998).

${ }^{8}$ G. Mie, Ann. Phys. (Leipzig) 25, 377 (1908); C. F. Bohren and D. R. Huffman, Absorption and Scattering of Light by Small Particles (J. Wiley, New York, 1983).

${ }^{9}$ K. S. Yee, IEEE Trans. Antennas and Propagation 14, 302 (1966).

${ }^{10}$ Allen Taflove, Computational Electrodynamics: The Finite-Difference Time-Domain Method (Artech House, Boston, 1995).

11 J. B. Pendry and A. MacKinnon, Phys. Rev. Lett. 69, 2772 (1992).

12 C. T. Chan, Q. L. Yu, and K. M. Ho, Phys. Rev. B 51, 16635 (1995).

${ }^{13}$ Kazuaki Sakoda and Hitomi Shiroma, Phys. Rev. B 56, 4830 (1997). 


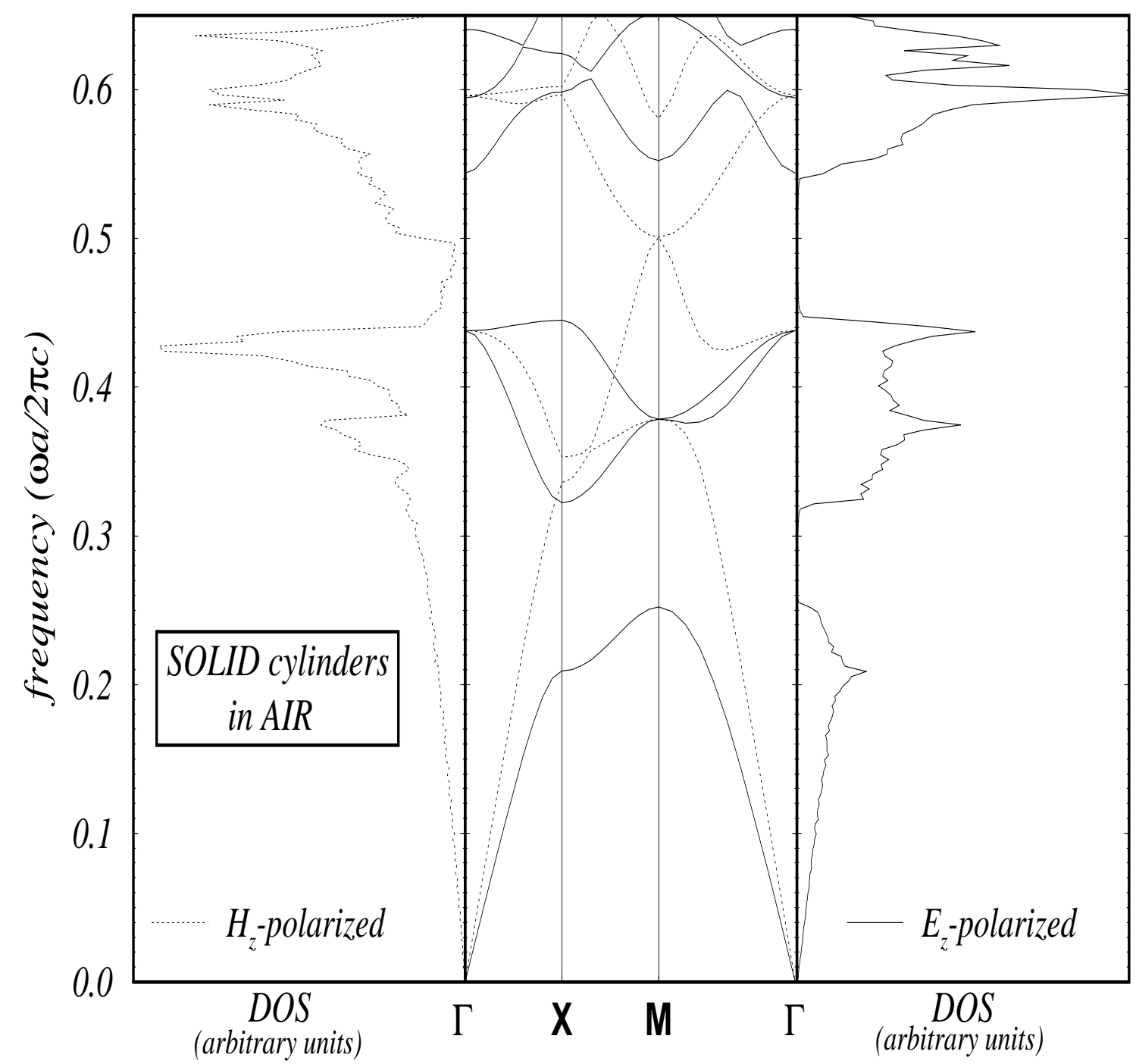

FIG. 1. Band structure (obtained with a plane wave method) and density of states (obtained with the FDTD spectral method) for a two-dimensional square lattice array of dielectric cylinders $\epsilon_{a}=10$ in air $\epsilon_{b}=1$, with a filling ratio $f \simeq 28 \%$. 


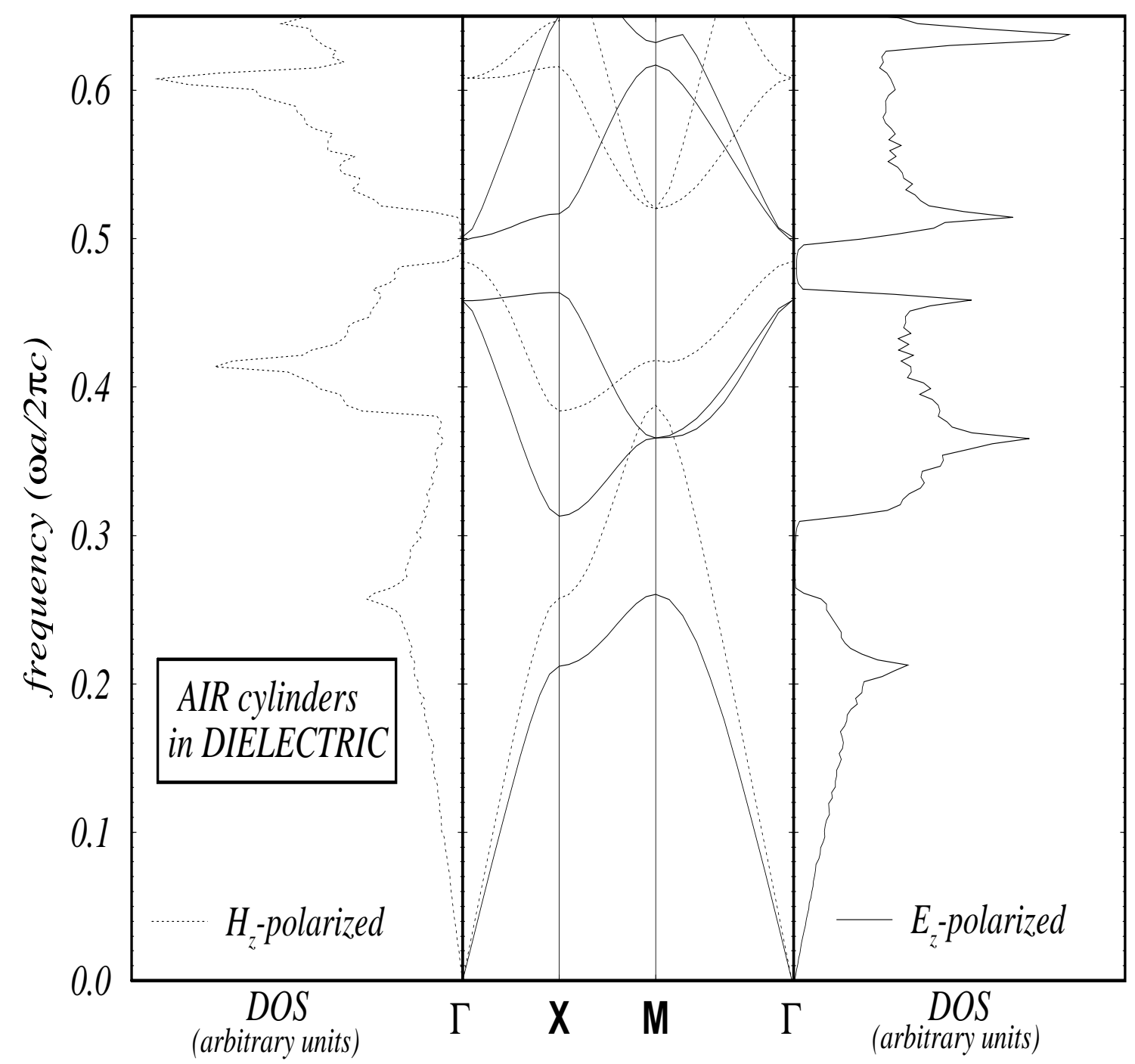

FIG. 2. Band structure (obtained with a plane wave method) and density of states (obtained with the FDTD spectral method) for a two-dimensional square lattice array of air cylinders $\epsilon_{a}=1$ in dielectric $\epsilon_{b}=10$, with air filling ratio $f \simeq 71 \%$. 


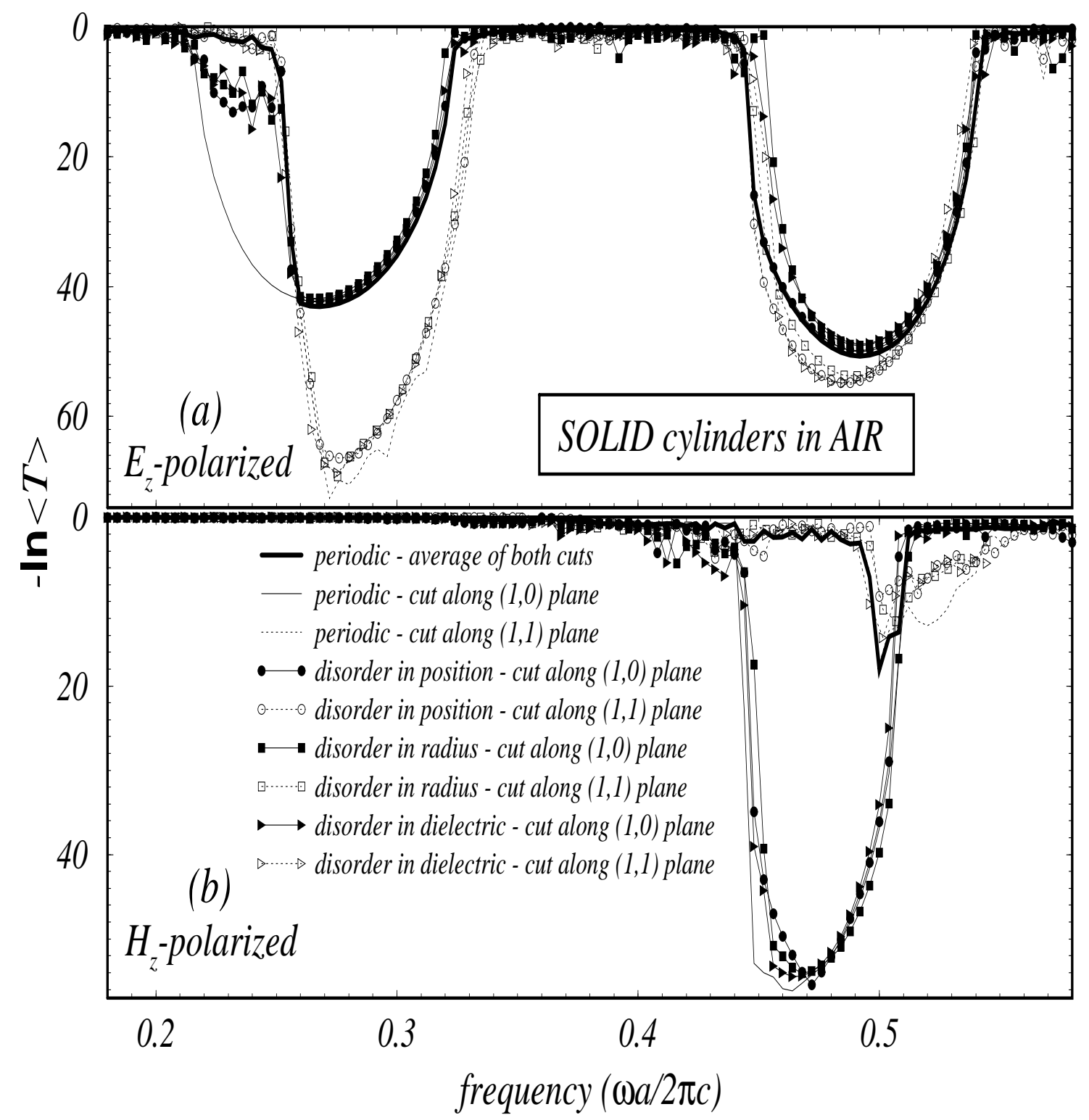

FIG. 3. Transmission coefficient for the periodic, and weakly disorder, system described in Fig. 1 (obtained with the transfer matrix technique). Calculations are for two different surfaces along which the sample is cut. Effective disorders used (look Eq. (4) $)$ : in position $\sim 1.3$, in radius $\sim 0.5$, and in dielectric $\sim 0.3$. 


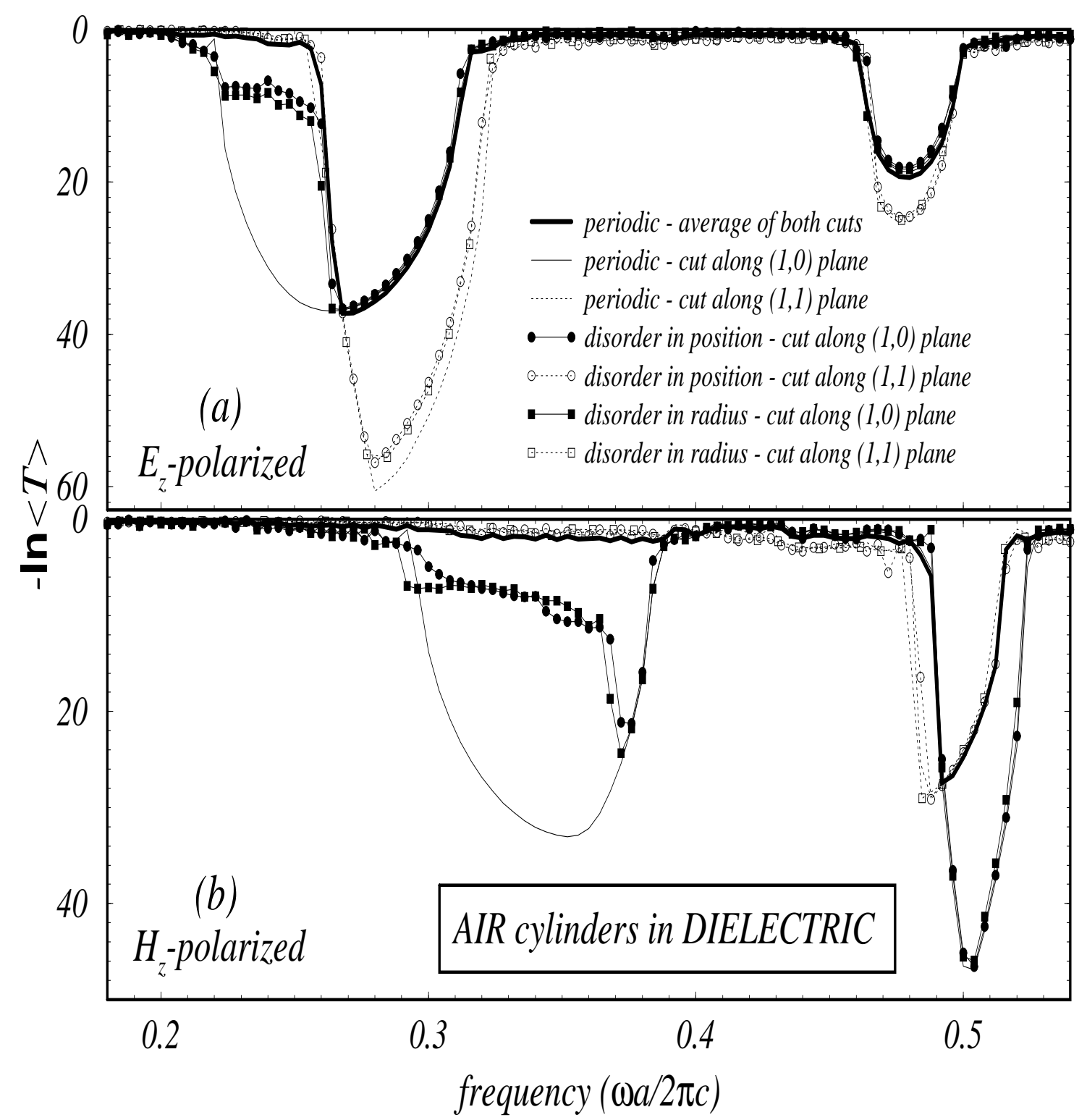

FIG. 4. Transmission coefficient for the periodic, and weakly disorder, system described in Fig. 2 (obtained with the transfer matrix technique). Calculations are for two different surfaces along which the sample is cut. Effective disorders used: in position $\sim 0.35$, in radius $\sim 0.25$ 


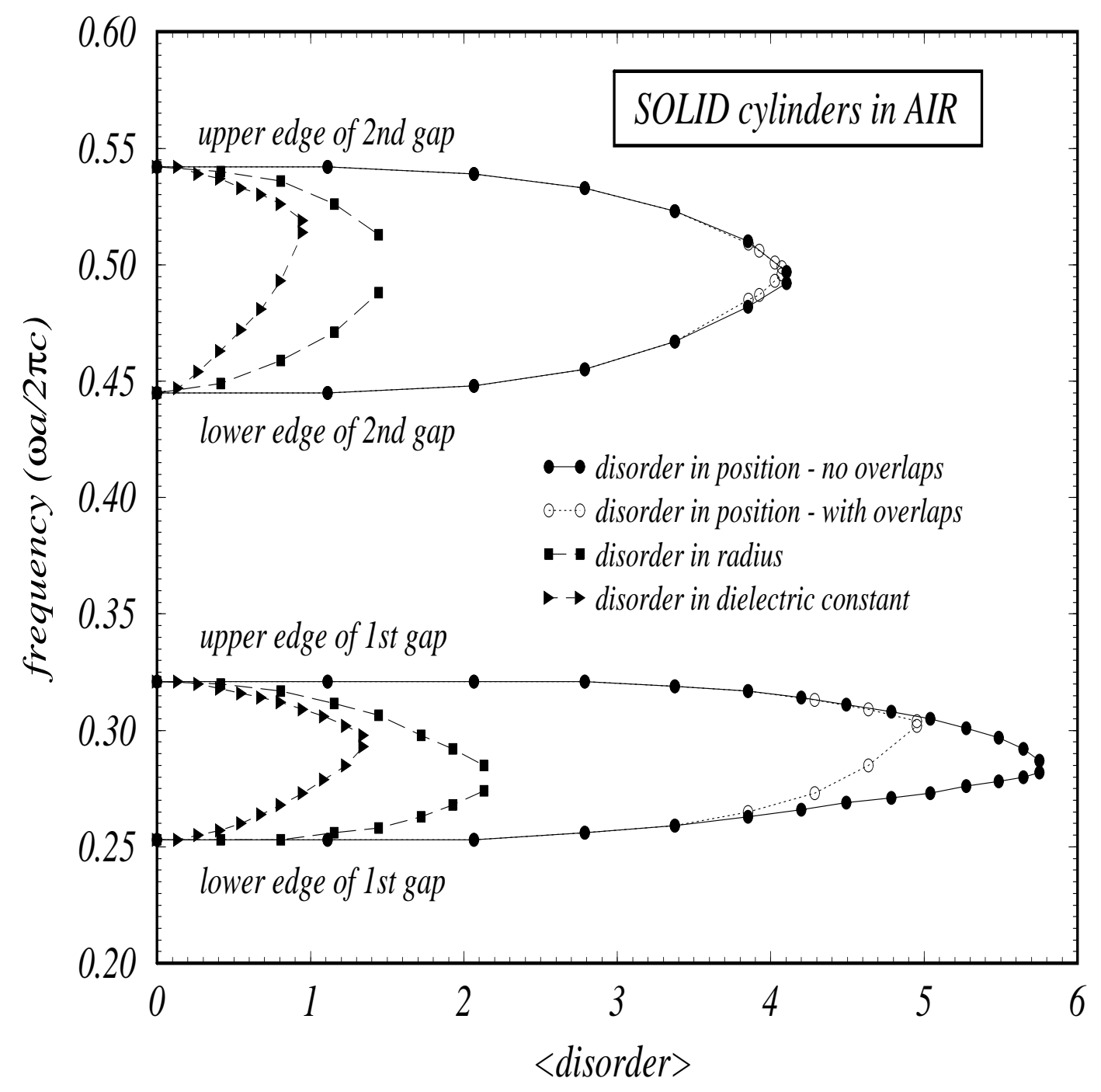

FIG. 5. The edges of the photonic band gaps as a function of the effective disorder $\langle$ disorder $>\equiv<\epsilon\rangle$ (as was defined in Eq. (4) ), for the system described in Fig. 1. Four different disorder realizations are studied. 


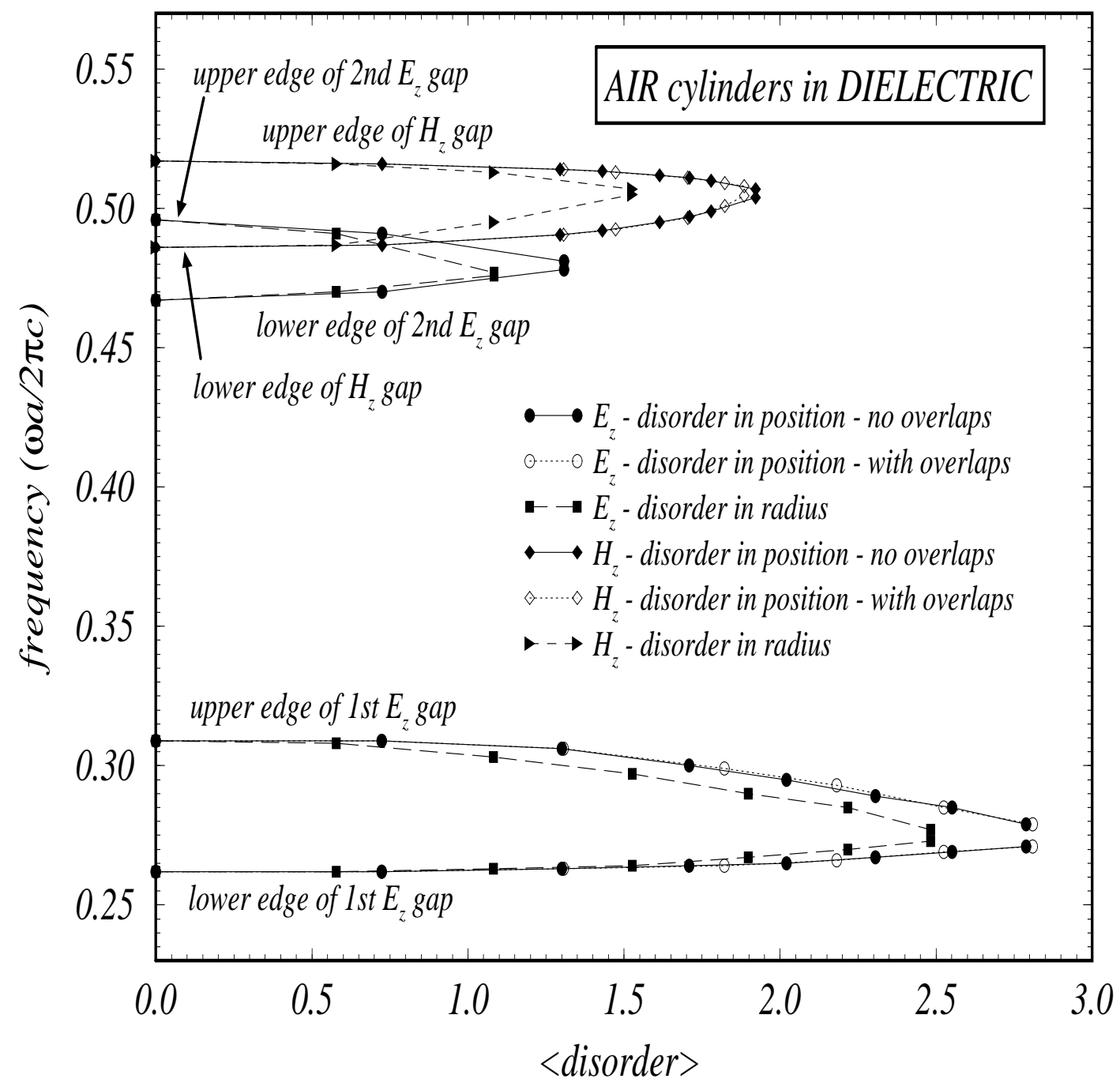

FIG. 6. The edges of the photonic band gaps as a function of the effective disorder $\langle$ disorder $>\equiv<\epsilon>$ (as was defined in Eq. (4)), for the system described in Fig. 2. Three different disorder realizations are studied. 


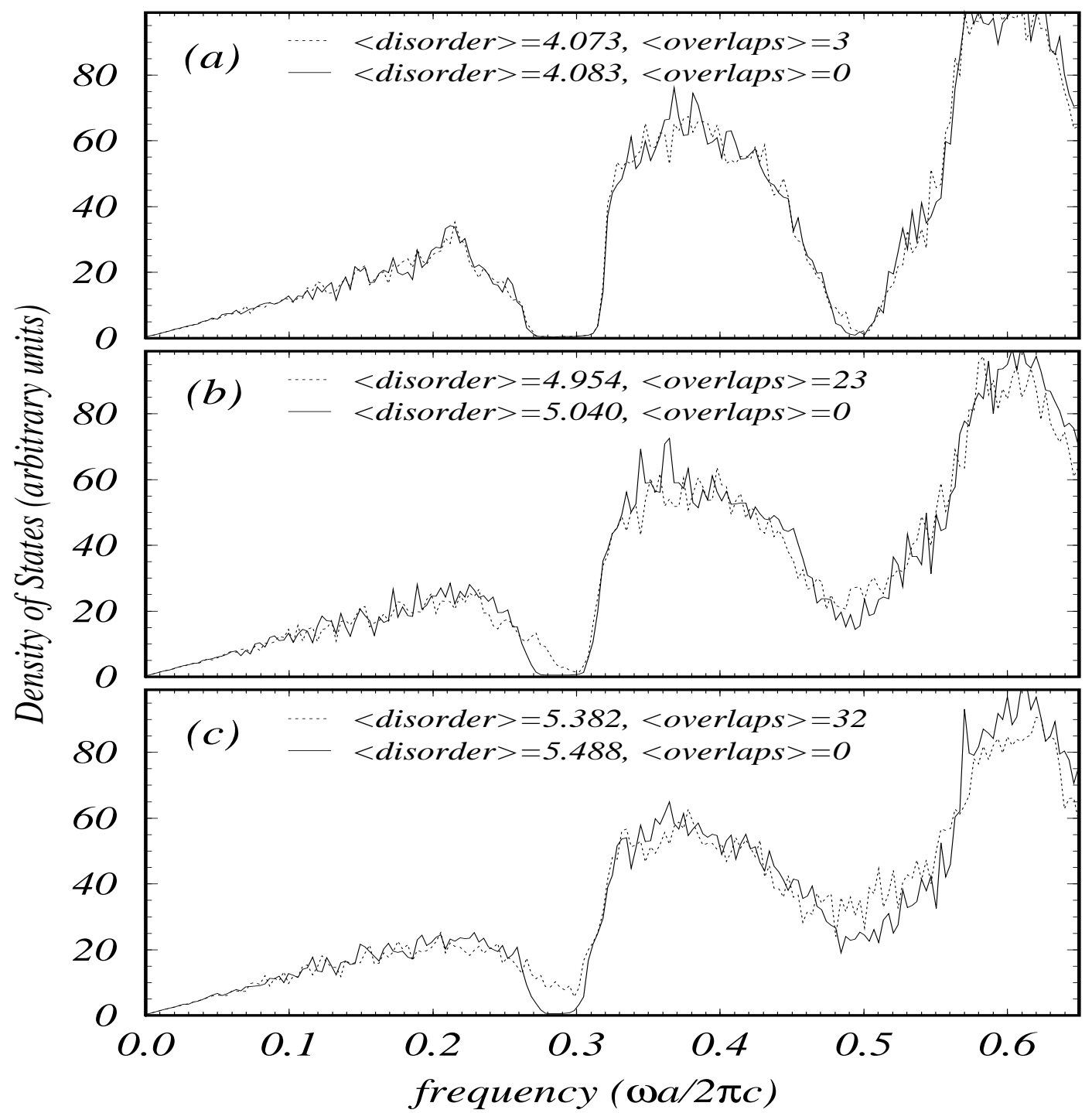

FIG. 7. The density of states for the system of Fig. 1 with the $E_{z}$ polarization, for three different positional disorder strengths. The solid line is when no scatterer overlaps are allowed, while the dotted line is when scatterer overlaps are allowed. $<$ overlaps $>$ is the average number of overlapping cylinders. 


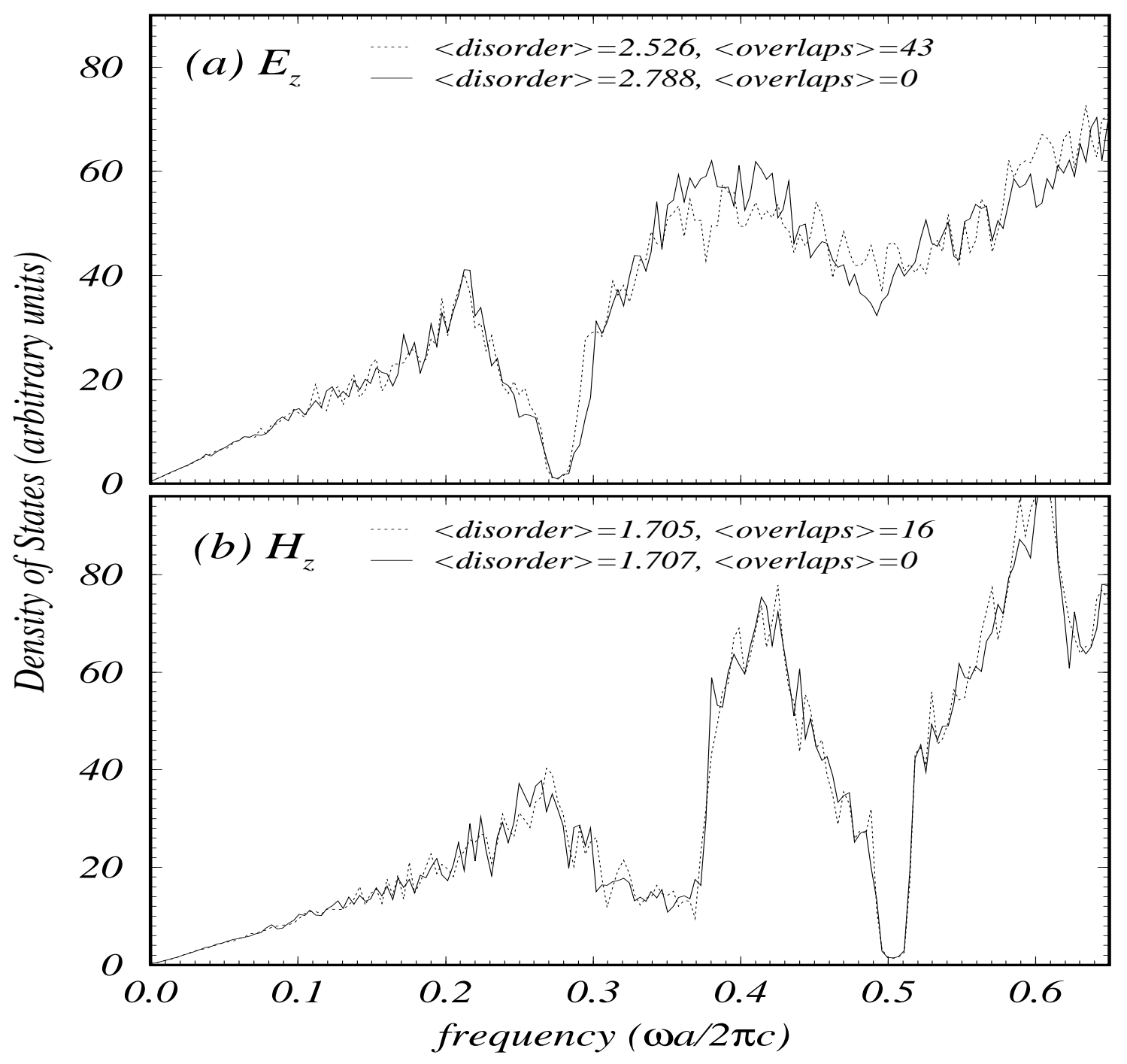

FIG. 8. The density of states for the system of Fig. 2 for both field polarizations, for two different positional disorder strengths. The solid line is when no scatterer overlaps are allowed, while the dotted line is when scatterer overlaps are allowed. 


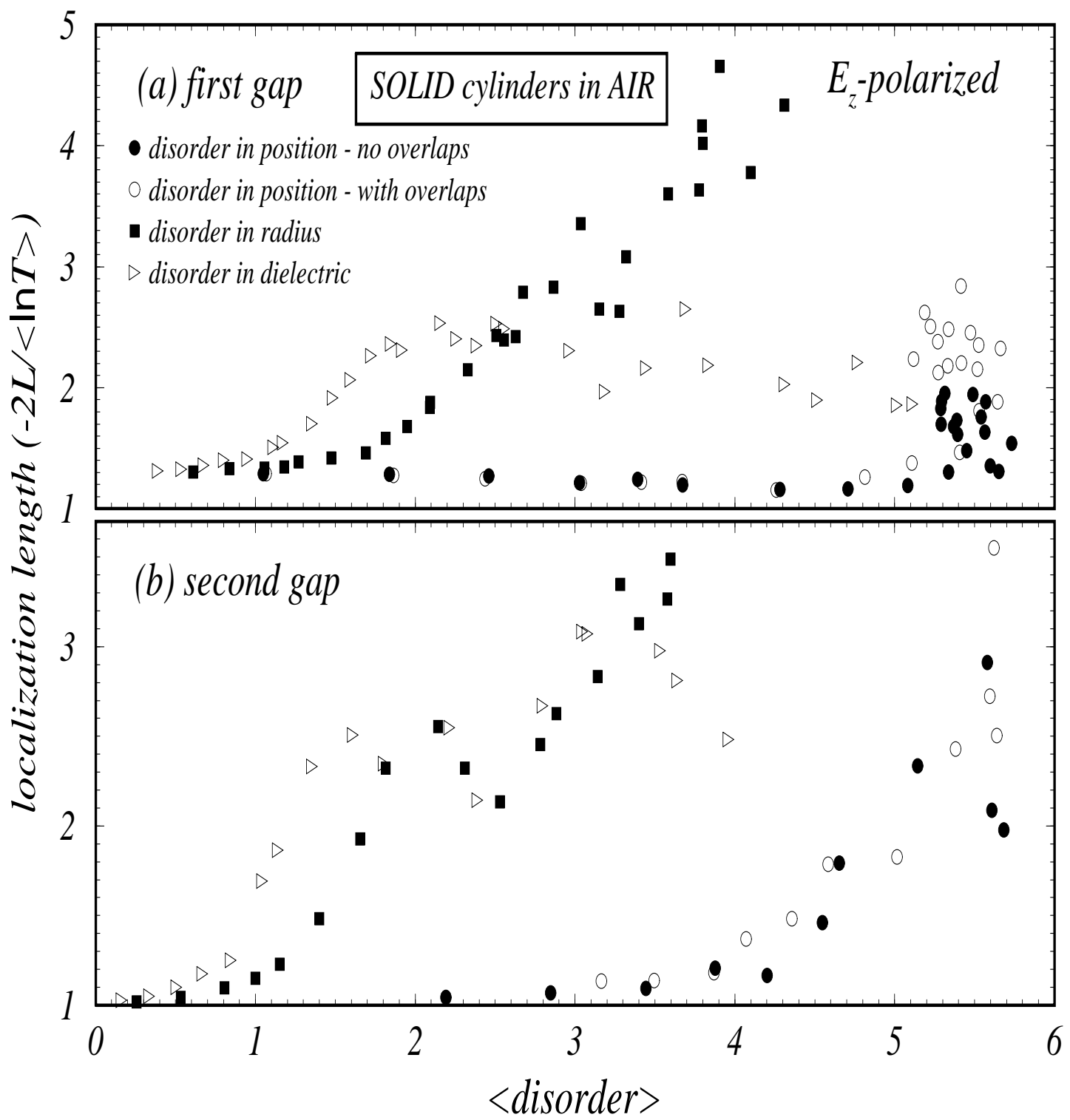

FIG. 9. The localization length as a function of the effective disorder for the system described in Fig. 1 with the $E_{z}$ polarization, for four different disorder realizations. 


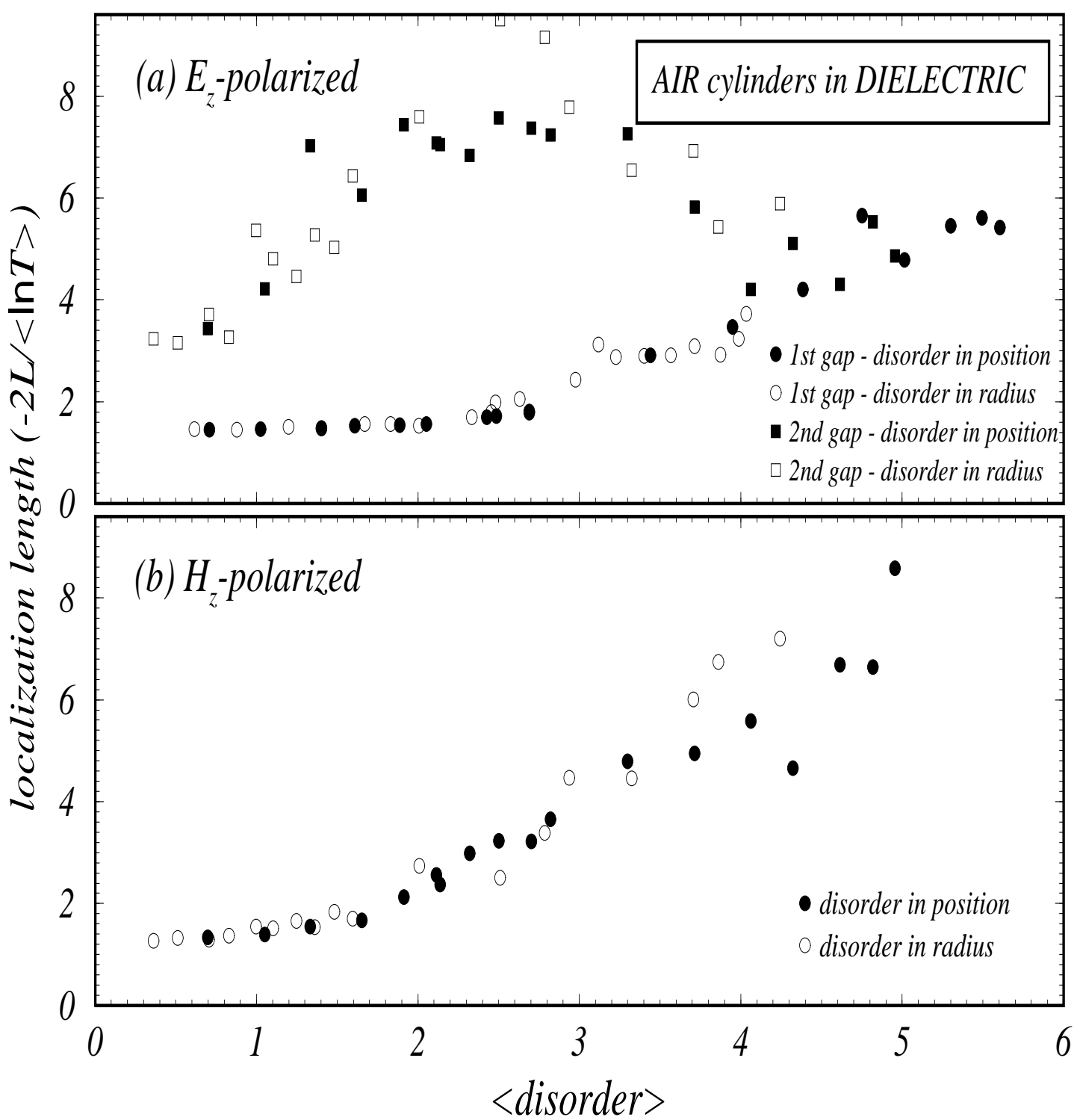

FIG. 10. The localization length as a function of the effective disorder for the system described in Fig. 2 for both field polarizations, for two different disorder realizations. 